\title{
Second Primary Malignancies in Chronic Myeloid Leukemia
}

\author{
Binay Kumar Shah $\cdot$ Krishna Bilas Ghimire
}

Received: 16 November 2013/Accepted: 27 December 2013/Published online: 23 January 2014

(C) Indian Society of Haematology \& Transfusion Medicine 2014

\begin{abstract}
Survival of patients with chronic myeloid leukemia (CML) has improved with the use of imatinib and other tyrosine kinase inhibitors. There is limited data on second primary malignancies (SPM) in CML. We analyzed the SPMs rates among CML patients reported to Surveillance, Epidemiology, and End Results (SEER) database during pre-(1992-2000) and post-(2002-2009) era. We used SEER Multiple Primary-Standardized Incidence Ratio session to calculate standardized incidence ratios (SIRs). Among 8,511 adult CML patients, 446 patients developed 473 SPMs. The SIR for SPMs in CML patients was significantly higher with observed/expected ratio:1.27, $P<0.05$ and absolute excess risk of 32.09 per 10,000 person years compared to general population. The rate of SPMs for cancers of all sites in post-imatinib era were significantly higher compared to pre-imatinib era with observed/expected ratio of 1.48 versus $1.06, P=0.03$. This study showed that risk of SPMs is higher among CML patients. The risk of SPMs is significantly higher in postimatinib era compared to pre-imatinib era.
\end{abstract}

Keywords CML $\cdot$ Imatinib $\cdot$ Second primary malignancies $\cdot$ SEER

This paper was presented at 2012 American Society of Hematology Annual Meeting and Exposition, Atlanta, GA, USA.

B. K. Shah $(\bowtie)$

Cancer Center and Blood Institute, St. Joseph Regional Medical Center, 1250 Idaho Street, Lewiston, ID 83501, USA

e-mail: binay.shah@gmail.com

K. B. Ghimire

Mercy Medical Center - North Iowa, Mason City, IA, USA

\section{Introduction}

Chronic myeloid leukemia (CML) is a hematopoietic stem cell disorder accounting for $15 \%$ of adult leukemias. It is characterized by translocation $\mathrm{t}(9 ; 22)$ resulting in the fusion gene (bcr-abl) product which is central to the development of the malignancy. Imatinib, which selectively inhibits bcrabl tyrosine kinase in CML patients, was approved by Food and Drug Administration in May 2001 based on the findings of International Randomized Study of Interferon and ST1571 trial [1]. In this pivotal trial, 1,106 newly diagnosed CML patients were randomized to receive either imatinib $400 \mathrm{mg}$ a day or interferon alpha plus low dose cytarabine. Results of this trial showed that imatinib induced major/ complete cytogenetic responses in $>85 \%$ of the patients and was associated with a relapse-free survival and overall survival of approximately 80 and $95 \%$, respectively. Because of high risk of relapse after stopping imatinib, or second generation TKIs such as nilotinib or desatinib, current recommendation is to continue the treatment with these drugs indefinitely outside the context of clinical trials. A potential immunosuppressive effect of imatinib was previously reported [2]. If this finding may lead to an increased risk of cancer in humans is still unknown [3]. In addition, a preclinical study showed the carcinogenic potential of imatinib [4]. We conducted a population based study utilizing The Surveillance, Epidemiology, and End Results (SEER) database to evaluate the incidence of SPM in CML patients in pre- and post- imatinib eras.

\section{Methodology}

SEER program from the National Cancer Institute is a population-based cancer registry that covers $26 \%$ of the 
Table 1 SIRs and AER for second primary malignancies among CML patients from 1992-2009 (person: 8,511, person years: $31,614.37)$
* excess risk per 10,000

\# $P<0.05$

\begin{tabular}{|c|c|c|c|c|}
\hline Secondary cancers & Observed & $\mathrm{O} / \mathrm{E}$ & CI $(95 \%)$ & Excess risk" \\
\hline All sites & 473 & $1.27 \#$ & $1.16-1.39$ & 32.09 \\
\hline All sites excluding non-melanoma skin & 470 & $1.27 \#$ & $1.16-1.39$ & 31.64 \\
\hline All solid tumors & 375 & $1.14 \#$ & $1.02-1.26$ & 14.14 \\
\hline Oral cavity and pharynx & 23 & $2.73 \#$ & $1.73-4.09$ & 4.61 \\
\hline Digestive system & 97 & $1.29 \#$ & $1.05-1.58$ & 6.95 \\
\hline Esophagus & 3 & 0.72 & $0.15-2.09$ & -0.38 \\
\hline Stomach & 10 & 1.38 & $0.66-2.54$ & 0.87 \\
\hline Small intestine & 2 & 1.34 & $0.16-4.85$ & 0.16 \\
\hline Colon, rectum and anus & 54 & 1.26 & $0.94-1.64$ & 3.49 \\
\hline Liver, gallbladder, intrahep bile duct and other biliary & 15 & $1.89 \#$ & $1.06-3.11$ & 2.23 \\
\hline Pancreas & 12 & 1.2 & $0.62-2.09$ & 0.62 \\
\hline Respiratory system & 72 & 1.27 & $0.99-1.6$ & 4.82 \\
\hline Lung, bronchus, trachea, mediastinum and other resp org & 65 & 1.23 & $0.95-1.56$ & 3.78 \\
\hline Soft tissue including heart & 2 & 1.02 & $0.12-3.7$ & 0.02 \\
\hline Skin excluding basal and squamous & 20 & 1.32 & $0.81-2.04$ & 1.55 \\
\hline Breast & 34 & 0.88 & $0.61-1.23$ & -1.51 \\
\hline Female breast & 33 & 0.86 & $0.59-1.21$ & -1.66 \\
\hline Male breast & 1 & 1.94 & $0.05-10.79$ & 0.15 \\
\hline Female genital system & 11 & 0.74 & $0.37-1.33$ & -1.2 \\
\hline Cervix uteri & 3 & 1.74 & $0.36-5.1$ & 0.4 \\
\hline Corpus and uterus, nos & 4 & 0.52 & $0.14-1.33$ & -1.17 \\
\hline Ovary & 3 & 0.72 & $0.15-2.11$ & -0.36 \\
\hline Male genital system & 70 & 0.9 & $0.7-1.13$ & -2.53 \\
\hline Prostate & 67 & 0.87 & $0.68-1.11$ & -3.05 \\
\hline Urinary system & 32 & 1 & $0.68-1.41$ & 0.01 \\
\hline Urinary bladder & 17 & 0.82 & $0.48-1.32$ & -1.16 \\
\hline Kidney and renal pelvis & 13 & 1.24 & $0.66-2.13$ & 0.81 \\
\hline Brain and other nervous system & 5 & 1.28 & $0.42-2.99$ & 0.35 \\
\hline Endocrine system & 8 & 1.72 & $0.74-3.4$ & 1.06 \\
\hline Thyroid & 6 & 1.4 & $0.51-3.05$ & 0.54 \\
\hline Adrenal gland & 2 & $16.90 \#$ & $2.05-61.04$ & 0.6 \\
\hline All lymphatic and hematopoietic diseases & 78 & $2.49 \#$ & $1.97-3.11$ & 14.78 \\
\hline Lymphoma & 40 & $2.43 \#$ & $1.74-3.31$ & 7.45 \\
\hline Myeloma & 5 & 1 & $0.32-2.33$ & 0 \\
\hline Leukemia & 33 & $3.37 \#$ & $2.32-4.73$ & 7.34 \\
\hline Mesothelioma & 2 & 1.9 & $0.23-6.86$ & 0.3 \\
\hline Kaposi sarcoma & 2 & 3.47 & $0.42-12.53$ & 0.45 \\
\hline Miscellaneous & 17 & 2.02\# & $1.18-3.24$ & 2.72 \\
\hline
\end{tabular}

United States population. Cancer incidence and survival data from 18 geographic areas in the US are collected in the SEER program [5]. We searched the SEER database: Incidence-SEER 13 Regs Research Data, November 2011 Sub, Vintage 2009 Pops (1992-2009) for adult patients with CML using Multiple Primary-Standardized Incidence Ratio (MP-SIR) Session. SEER 13 covers approximately $13.8 \%$ of US population. [Geographic areas and years covered in SEER 13 registry are: San Francisco-Oakland SMSA, 1992+; Connecticut, 1992+; Detroit (Metropolitan),
1992+; Hawaii, 1992+; Iowa, 1992+; New Mexico, 1992+, Seattle (Puget Sound), 1992+, Utah, 1992+; Atlanta (Metropolitan), 1992+; San Jose-Monterey, 1992+; Los Angeles, 1992+, Alaska Natives, 1992+, Rural Georgia, 1992+]. We analyzed second cancer rates among adult CML patients during the period 1992-2009, 1992-2000 (pre-imatinib era) and 2002-2009 (post-imatinib era) using SEER-Stat, a statistical software provided by NCI for statistical analysis. We used SEER MP-SIR session and Graph pad scientific software to calculate second primary 


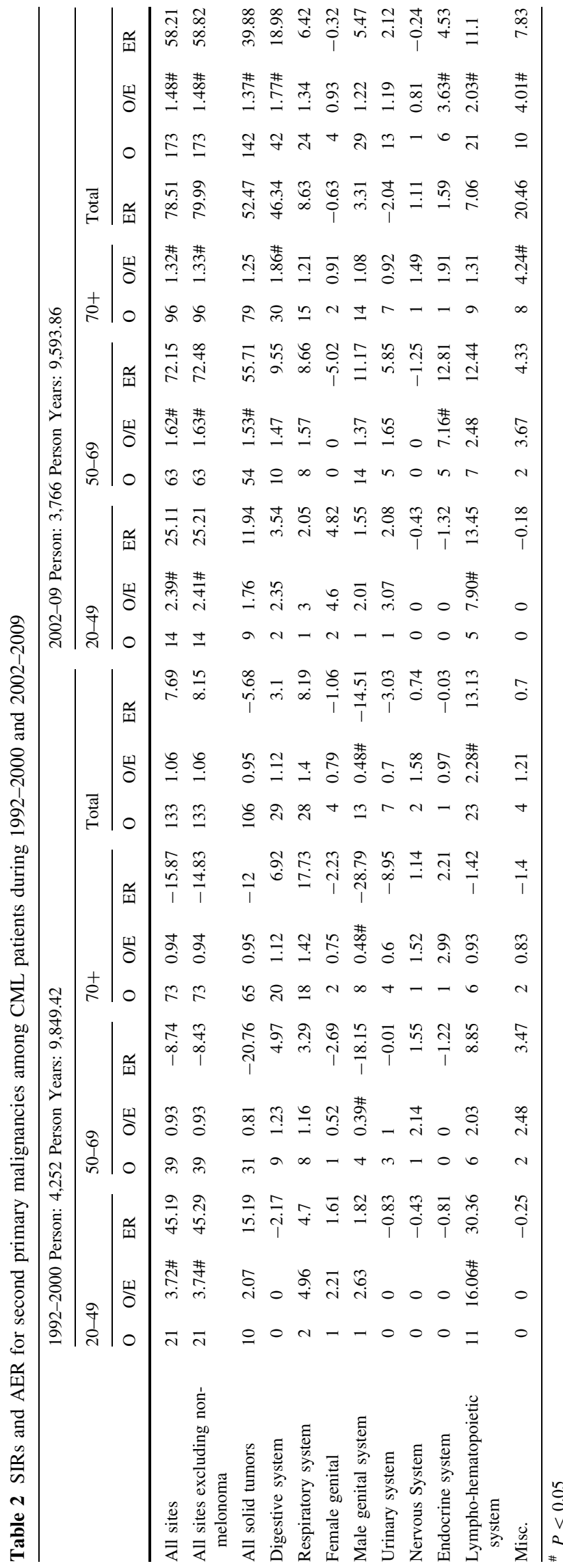

malignancies in CML patients during 1992-2009, 1992-2000 and 2002-2009 time periods. The patient was followed from 1 month after diagnosis of CML to date of last known vital status, death, or end of the study (31 December 2009) which ever come first. CML patients diagnosed during 2001 were excluded from the study to avoid overlapping in two groups of patients studied.

\section{Results}

The total number of adult CML patients (age older than 20 years) reported during $1992-2009$ period was 8,511 . The study population included 4,979 men and 3,532 women. Median age at the time of diagnosis was 70.8 years (Range 25.08-95.92 years).

\section{Second Primary Malignancies in CML Patients}

Among these patients, 473 cases of secondary primary cancer were found in 446 patients, and 22 patients with CML developed more than one second primary cancer. The rate of second primary cancer was significantly higher among CML patients compared to that expected in the general population with observed/expected ratio $(\mathrm{O} / \mathrm{E}): 1.27,95 \%$ confidence interval (CI), 1.16-1.39; $P<0.05$; absolute excess risk (AER): 32.09 per 10,000 person years. The risk of second cancer was higher for all solid tumor, oral cavity and pharynx, digestive system, liver and biliary, adrenal, lymphatic and hematological cancer (Table 1).

Secondary Primary Malignancies in Pre- and PostImatinib Era

There were 4,252 and 3,766 CML patients in the pre(1992-2000) and post- (2002-2009) Imatinib era respectively. The total person years at risk was $9,849.42$ for preimatinib era and 9,593.86 years for post-imatinib era. The total numbers of SPMs were 133 (129 patients) in preimatinib and 173 (167 patients) in post-imatinib era (Table 2). The median age at the time of diagnosis of SPM was, 71 years (rage: $27.67-91.67$ years) and 71.58 years (range: 26.17-93.0 years). The median follow up time since the diagnosis of CML was 19 months (range 1-85 months) and 13 months (range 1-91 months) in preand post- imatinib era respectively.

The rate of SPMs for cancers of all sites in post-imatinib era was significantly higher compared to pre-imatinib era with observed/expected $(\mathrm{O} / \mathrm{E})$ ratio of 1.06 versus 1.48 , $P=0.0007$. The AERs for cancers of all sites in pre-imatinib and post-imatinib era were 7.69 and 58.21 respectively. Similarly, the rates of SPMs for cancers of all sites excluding non-melanoma skin cancer were significantly 
higher in post-imatinib era compared to pre-imatinib era with $\mathrm{O} / \mathrm{E} 1.06$ versus $1.48, P=0.0007$ respectively. The rate of SPMs for all solid tumor was significantly higher in post-imatinib era compared to pre-imatinib era with $\mathrm{O} / \mathrm{E}$ 0.95 versus $1.37, P=0.0012$. Digestive system cancer was more common in post imatinib era with $\mathrm{O} / \mathrm{E} 1.12$ versus $1.77 P=0.04$.

\section{Analysis by Sex}

Among patients who developed SPM, male comprised of 78 $(60.4 \%)$ in pre imatinib era and $109(65.27 \%)$ post-imatinib era. Median age at SPM for male was 70.58 years (range: 27.67-89.58 years) and 71.5 years (range: 26.17-93 years) in pre- and post- imatinib era respectively. Similarly, for female, median ages at SPM were 74 years (range: $32.17-91.67$ years) and 72.29 years (range: $40.08-91.75$ years) in pre- and postimatinib era respectively.

\section{Anylasis by Age Groups}

We analysed SPM rates by age groups 20-49, 50-69, and $70+$ years during both pre- and post- imatinib era. For patients aged 50-69 years, all sites SPM was significantly increased in post-imatinib era as compared to pre-imatinib era with $\mathrm{O} / \mathrm{E}$ ratio $0.93(n=39)$ versus $1.62(n=63)$, $P=0.0027$, and all solid tumor $\mathrm{O} / \mathrm{E}$ ratio $0.81(n=31)$ versus $1.53(n=4), P=0.002$. All site $\mathrm{SPM}$ was also increased in $70+$ years age group patients in post-imatinib era with $\mathrm{O} / \mathrm{E}$ ratio $0.94(n=73)$ versus $1.32(n=96)$, $P=0.01$ (Table 2). There was also increase in $\mathrm{O} / \mathrm{E}$ ratio and excess risk of liver and biliary, melanoma, endocrine SPM in 50-69 years age group in post-imatinib era but the increase was not statistically significant as compared to pre-imatinib era. Similarly, digestive system tumor O/E ratio and excess risk increased in $70+$ age groups in post-imatinib era but it was not statistically as compared to pre-imatinib era.

All site tumor was increased in post-imatinib era 50-69 years age group, $P=0.0027$, and $70+$ years age group, $P=0.01$. All solid tumor was significantly increased in 50-69 years age group, $P=0.002$.
Latency to the Development of Second Primary Malignancy

Patients were followed for median time of 19 months (range 1-85 months) and 13 months (range 1-91 months) in pre- and post- imatinib era respectively. The risk of second primary cancer was highest within 1-11 months for all age groups in post-imatinib era (Table 3 ).

\section{Discussion}

Improvement in survival of CML is a success story and is largely due to approval of tyrosine kinase inhibitors. Our previous population based study [6] showed that the survival benefit has translated to population based settings.

This is the largest study to evaluate second primary cancers in patients with CML. This study showed that overall risk of second malignancies among CML patients is higher compared to general population. There is significantly higher risk for SPM of digestive system, liver and biliary, adrenal, lymphatic and hematological malignancy. A recent study from Europe [7] showed slight increase in secondary malignancies in CML patients compared to general population. In this study, the age standardized incidence rates of secondary malignancies in CML patients were 535 and 582 per 100,000 for men and women respectively. The incidence rates for general population in Germany were 450 and 350 per 100,000 for men and women. These findings are important in view of improved survival among CML patients. These patients may benefit from regular examination for other malignancies during their follow up visits.

Interestingly, the overall risk of second primary malignancies is significantly higher in post-imatinib era. However, there was no significant difference in risk of specific SPMs. This may be because of rarity of this late complication. In a 2 year rat carcinogenicity study [8] that included administration of imatinib at 15, 30 and $60 \mathrm{mg} / \mathrm{kg} /$ day showed neoplastic changes in renal tubule, renal pelvis, urinary bladder, urethra, preputial and clitoral

Table 3 SPM latency during 1992-2000 and 2002-2009

\begin{tabular}{|c|c|c|c|c|c|c|c|c|c|c|c|c|c|c|c|c|}
\hline & \multicolumn{8}{|c|}{$1992-2000$} & \multicolumn{8}{|c|}{ 2002-2009 } \\
\hline & \multicolumn{2}{|c|}{ 20-49 years } & \multicolumn{2}{|c|}{$50-69$ years } & \multicolumn{2}{|c|}{$70+$ years } & \multicolumn{2}{|l|}{ Total } & \multicolumn{2}{|c|}{ 20-49 years } & \multicolumn{2}{|c|}{$50-69$ years } & \multicolumn{2}{|c|}{$70+$ years } & \multicolumn{2}{|c|}{ Total } \\
\hline & $\mathrm{O}$ & $\mathrm{O} / \mathrm{E}$ & $\mathrm{O}$ & $\mathrm{O} / \mathrm{E}$ & $\mathrm{O}$ & $\mathrm{O} / \mathrm{E}$ & $\mathrm{O}$ & $\mathrm{O} / \mathrm{E}$ & $\mathrm{O}$ & $\mathrm{O} / \mathrm{E}$ & $\mathrm{O}$ & $\mathrm{O} / \mathrm{E}$ & $\mathrm{O}$ & $\mathrm{O} / \mathrm{E}$ & $\mathrm{O}$ & $\mathrm{O} / \mathrm{E}$ \\
\hline $1-11$ months & 2 & 1.22 & 16 & 1.23 & 23 & 0.84 & 41 & 0.97 & 5 & $3.23 \#$ & 20 & $1.80 \#$ & 53 & $2.14 \#$ & 78 & 2.08\# \\
\hline $12-59$ months & 14 & $4.26 \#$ & 19 & 0.75 & 43 & 0.97 & 76 & 1.04 & 9 & $2.43 \#$ & 36 & $1.51 \#$ & 40 & 0.94 & 85 & 1.21 \\
\hline 60-119 months & 5 & $6.88 \#$ & 4 & 1.11 & 7 & 1.15 & 16 & 1.54 & 0 & 0 & 7 & 1.79 & 3 & 0.59 & 10 & 1.04 \\
\hline Total & 21 & 3.72\# & 39 & 0.93 & 73 & 0.94 & 133 & 1.06 & 14 & 2.39\# & 63 & $1.62 \#$ & 96 & $1.32 \#$ & 173 & $1.48 \#$ \\
\hline
\end{tabular}

\# $P<0.05$ 
glands, small intestine, parathyroid glands, adrenal glands, and non glandular stomach. Neoplastic changes were not observed at $30 \mathrm{mg} / \mathrm{kg} /$ day for the kidneys, urinary bladder, urethra, small intestine, parathyroid glands, adrenal glands and non-glandular stomach, and at $15 \mathrm{mg} / \mathrm{kg} / \mathrm{day}$ for preputial and clitoral gland.

A previous study [9] did not show any significant increase in risk of secondary cancers from exposure to tyrosine kinase inhibitors. In this study, records of 1,445 patients with CML/ Myeloproliferative neoplasm and other hematologic malignancies treated with TKIs were reviewed. The risk of second cancer was found to be lower than expected risk with observed to expected ratio $0.6,95 \%$ confidence interval 0.44-0.81. This study however, did not include CML patients only. In contrast, our study included larger number of patients with CML only. Second primary malignancy is a rare event and relatively smaller sample size in the study by Verma D et al. [9] may be responsible for lack of association between imatinib and SPMs.

The increase in SPMs in CML patient in post-imatinib era is unexplained. The use of tyrosine kinase for treatment of CML may be responsible for significant increase in second primary malignancies.

Another potential explanation of increased SPMs rate in post-imatinib era may be inherent higher risk of SPMs among CML patients. Since survival of CML patients has improved with use of TKI, more SPMs are being diagnosed in these patients.

It may be important to screen patients with CML for second primary malignancies during follow up visits. This is specifically more important in older patients and during the first year of diagnosis of CML.

\section{Conclusion}

Risk of second primary malignancies is significantly higher in patients with CML. SPM rate has significantly increased during post-imatinib era compared to pre-imatinib era.

\section{References}

1. O'Brien SG, Guilhot F, Larson RA, Gathmann I, Baccarani M, Cervantes $F$ et al (2003) Imatinib compared with interferon and low-dose cytarabine for newly diagnosed chronic-phase chronic myeloid leukemia. N Engl J Med 348(11):994-1004

2. Seggewiss R, Loré K, Greiner E, Magnusson MK, Price DA, Douek DC et al (2005) Imatinib inhibits T-cell receptor-mediated $\mathrm{T}$-cell proliferation and activation in a dose-dependent manner. Blood 105:2473-2479

3. Penn I, Starzl TE (1973) Immunosuppression and cancer. Transplant Proc 5(1):943-947

4. http://www.pharma.us.novartis.com/product/pi/pdf/gleevec_tabs.pdf. Accessed 21 Sept 2013

5. http://seer.cancer.gov/about/factsheets/SEER_Research_Brochure. pdf. Accessed 21 Sept 2013

6. Mandal R, Bolt DM, Shah BK (2013) Disparities in chronic myeloid leukemia survival by age, gender, and ethnicity in preand post-imatinib eras in the US. Acta Oncol 52(4):837-841

7. Miranda MB, Lauseker M, Proetel U, Schreiber A, Hanfstein B, Baerlocher GM, et al. Secondary malignancies in CML patientsdata from the german CML study IV. 54th ASH Annual Meeting and Exposition; 8-11 December 2012. Abstr 3746

8. Imatinib prescribing information. Novartis Pharmaceuticals. 2010. http://www.pharma.us.novartis.com/product/pi/pdf/gleevec_ tabs.pdf. Accessed 21 Sept 2013

9. Verma D, Kantarjian H, Strom SS, Rios MB, Jabbour E, QuintasCardama A et al (2011) Malignancies occurring during therapy with tyrosine kinase inhibitors (TKIs) for chronic myeloid leukemia (CML) and other hematologic malignancies. Blood 118(16):4353-4358 